\title{
Evaluation Method of Complex Slope Stability in Construction Industry Based on Correlation Calculation
}

\author{
Weiwei Zhu \\ College of Architecture and Civil Engineering, Kunming University, Kunming, Yunnan, China
}

\begin{abstract}
Based on the basic-element expression method and correlation function theory in Extenics, combined with the knowledge of slope engineering, a method for evaluating the stability of complex slope is proposed. The evaluation process includes the selection of suitable evaluation indexes of slope stability, identification of the classification standard for the slope stability, identification of the weights of evaluation indexes by using the improved analytic hierarchy process, identification of the classical domain, node domain and matter-element under evaluation, the calculation of the correlation of each stability class for each evaluation index, normalization of correlation, and so on. The selection rule of the slop stability evaluation indexes and selection of correlation function and optimal points are also discussed, which can provide reference for slope engineering design in construction industry.
\end{abstract}

Keywords: construction industry; slope engineering, stability grade, Extenics, correlation

\section{Introduction}

The slope stability issue is an old and frequently updated subject in the engineering field. Plentiful slope stability analysis method has been proposed in the geotechnical engineering. The engineering technicians are familiar with the extreme balance method for quantitatively solving the slope stability security coefficient, but the applications of this method are limited and the slopes are diversified and the factors affecting the slope stability are very complex, so it is significant for applications to study quantitative and uncertain analysis methods. It also speeds up the applications of new theories and new methods in the slope stability analysis. The multi-discipline and multispecialty crossing and penetration research represents the new trend in the slope research.

The application of extension theory in slope engineering started late. Wang Liang ${ }^{[1]}$ first applied the Extenics theory into the stability analysis of the rock slope. Xie Quanmin ${ }^{[2]}$ proposes the extension clustering and prediction method of the rock slope stability. Li Kegang ${ }^{[3-6]}$ studies the relative importance of the evaluation indexes for determining slope stability based on the simple correlation function in the Extenics, proposes an improved analytic hierarchy process to solve the index weights, and proposes the complete slope stability analysis method; Tan Xiaolong ${ }^{[7]}$ establishes the whole security analysis method of the rock slope based on multiple levels and multiple indexes by using matter-element representation and correlation function.

On the basis of previous research results, based on the basic-element theory and correlation function theory of Extenics, combined with slope engineering knowledge, this paper provides a new effective method for the evaluation of complex slope stability.

\section{Extenics and Superiority Evaluation Method}

Extenics is an original trans-disciplinary science based on the philosophy, mathematics and engineering, which was proposed by Chinese researcher Cai Wen in 1983 and features formalization, logicalization and formalization ${ }^{[8]}$. The formalized models are used to discuss the thing extension possibility, explore the new laws and methods and apply them to solve contradiction problems in reality.

Superiority evaluation method is used to evaluate an object in Extenics, including superiority of things, strategies and methods. The superiority evaluation involves the following concepts:

ISSN: 0010-8189 


\subsection{Measurement indexes}

The first step for evaluating superiority of an object is to identify measurement indexes. Generally, the measurement index set is expressed as $S I=\left\{S I_{1}, S I_{2} \cdots, S I_{n}\right\}$ in the superiority evaluation, $S I_{i}=\left(c_{i}, V_{i}\right)$ is the characteristic element, $c_{i}$ is the evaluation feature and $V_{i}$ is the quantified measurement domain.

\subsection{Correlation}

For the object $Z$ under evaluation, the qualified measurement range is $X_{0}$ for a measurement index $S I$ and the permitted measurement range is $X$. The correlation function $K(z)$ indicates compliance of the object $Z$ to the requirements, which is called as the correlation of the $Z$ on $S I$.

\subsection{Specification correlation}

Given that the correlation of $Z$ to $S I$ is $K(z)$, then we can get:

$$
k_{Z}= \begin{cases}\frac{K(Z)}{\max _{x \in X_{0}} K(x)}, & K(z)>0 \\ \frac{K(Z)}{\max _{x \notin X_{0}}|K(z)|}, & K(z)<0\end{cases}
$$

It is called as the standardized correlation of $Z$ to $S I$.

\subsection{Superiority}

For the object $Z$ under evaluation, if the measurement index set is $S I=\left\{S I_{1}, S I_{2} \cdots, S I_{n}\right\}$, the standardized correlation of $Z$ to $S I_{i}$ is expressed as $k_{i}(i=1,2, \cdots n)$, the weight coefficient of $S I_{i}$ is $\alpha_{i}$, and $0 \leq \alpha_{i} \leq 1$, then

(1) When the comprehensive correlation of all measurement indexes is more than 0 , it indicates that the object $Z$ satisfies the requirement and the superiority is $C(Z)=\sum_{i=1}^{n} \alpha_{i} k_{i}$

(2) when the correlation of a measurement index is more than 0 , it indicates that the object $Z$ satisfies the requirement and the superiority is $C(Z)=\underset{i=1}{\stackrel{n}{V}} k_{i}$

(3) If the correlation of all measurement indexes is more than 0 , it indicates that this object $Z$ satisfies the requirement and the superiority is $C(Z)=\stackrel{n}{i=1}_{\wedge}^{n} k_{i}$

(4) If the correlation of a measurement index shall be more than or less than a threshold value $\lambda(\lambda>0)$, this index is first used to evaluate the evaluation object. The superiority of all objects satisfying this index will be calculated by using one of above three algorithms.

ISSN: 0010-8189 


\section{Steps of the Slope Stability Analysis Based on Superiority Evaluation Method}

\subsection{Identify evaluation indexes and classification standard of the slope stability}

The slope stabilities are controlled by plentiful complex factors. The slope stability shall be completely analyzed and considered in case of evaluation of slope. It is impossible and unnecessary to consider all influence factors. The decisive influence factors to destruction of slope shall be highlighted. The selected evaluation indexes are not consistent in current research, e.g. 10 factors such as terrain, rock integrity, basic rock quality index (BQ), weathering degree and slope angles are selected as the evaluation indexes in the reference ${ }^{[9]} .8$ factors such as lithology, gradient, slope structure, seismic intensity and rainfall are selected as the evaluation indexes in the reference $^{[10]}, 6$ evaluation factors such as the rock soil volume weight, cohesive strength, internal friction angle, gradient, slope height and hydraulic pressure ratio are selected as the evaluation indexes in the reference ${ }^{[11]} .22$ factors such as landslide shape characteristics, landslide history, landslide body structure, sliding surface features, hydrogeologic condition, landslide body structure and rainfall are selected as the evaluation index system in the reference $^{[12]}$. In addition, other evaluation indexes are diversified ${ }^{[13-17]}$, in a word, the selection rules of the evaluation indexes are summarized as follows:

(1)The nature and geological environment of the slope shall be completely summarized;

(2) The geometric shape features of the slope shall be completely reflected;

(3) The influences of the manmade activities on the slope stability shall be summarized.

In a word, the selected evaluation indexes shall focus on quality instead of quantity. The factors affecting the slope stability shall be highlighted and the selected evaluation indexes shall ensure easy calculation and reasonable weight assignation.

After the slope stability evaluation indexes are identified, the stability classification standards shall be identified. E.g. the slope stability is divided into sable, slightly stable, roughly stable, unstable and very unstable in the reference ${ }^{[18]}$. The classification standard for the slope stability is proposed in the reference ${ }^{[19]}$ based on summary of the past research achievements and national standards, shown as the table 1 .

Table 1 Classification standard of the slope stability

\begin{tabular}{|c|c|c|c|c|c|}
\hline Stability class & Class I & Class II & Class III & Class IV & Class V \\
\hline & stability & $\begin{array}{c}\text { slight } \\
\text { stability }\end{array}$ & $\begin{array}{c}\text { basic } \\
\text { stability }\end{array}$ & instability & $\begin{array}{c}\text { very } \\
\text { instability }\end{array}$ \\
\hline RQD & $>90$ & $90 \sim 75$ & $75 \sim 50$ & $50 \sim 25$ & $<25$ \\
\hline
\end{tabular}

ISSN: 0010-8189 


\begin{tabular}{|c|c|c|c|c|c|}
\hline $\begin{array}{c}\text { Rock body structure } \\
\text { characteristics }\end{array}$ & $>90$ & $90 \sim 75$ & $75 \sim 50$ & $50 \sim 30$ & $<30$ \\
\hline Crustal stress/MPa & $<2$ & $2 \sim 8$ & $8 \sim 14$ & $14 \sim 20$ & $>20$ \\
\hline Cohesive strength/KPa & $>220$ & $220 \sim 120$ & $120 \sim 80$ & $80 \sim 50$ & $<50$ \\
\hline Internal friction angle/ ${ }^{\circ}$ & $>37$ & $37 \sim 29$ & $29 \sim 21$ & $21 \sim 13$ & $<13$ \\
\hline Slope height/m & $<30$ & $30 \sim 45$ & $45 \sim 60$ & $60 \sim 80$ & $>80$ \\
\hline Gradient/ ${ }^{\circ}$ & $<15$ & $15 \sim 25$ & $25 \sim 35$ & $35 \sim 45$ & $>45$ \\
\hline Daily maximal rainfall /mm & $<20$ & $20 \sim 40$ & $40 \sim 60$ & $60 \sim 100$ & $>100$ \\
\hline Maximal seismic strength & $<3$ & $3 \sim 5$ & $5 \sim 7$ & $7 \sim 8$ & $>8$ \\
\hline
\end{tabular}

\subsection{Identify weights of evaluation indexes}

Different factors have difference influences on the slope stability. The traditional AHP (analytic hierarchy process) is mainly used to solve weights in existing research. When the AHP is used to solve weights, the key is to construct the judgement matrix. Once the judgement matrix is constructed incorrectly, the further calculation based on the judgement matrix is meaningless.

Doctor Li Kegang ${ }^{[5]}$ studied the influences of the dynamic index weights on the slope stability that thought that weights of the evaluation indexes are random and fuzzy and one index has different influences on the slope stability. Namely if the evaluation index is identified in weight solving based on the traditional AHP method, the experiences from several experts are used to determine the relative important of indexes in the evaluation system and give corresponding weights. This method focuses on selection of the evaluation indexes and ignores the fact that different values have different influence on the system. The judgement matrix is constructed based on sorting of index significance and the index weights are solved. It will not correctly reflect actual conditions and finally weakens reliability and practicability of the evaluation results. Based on it, Doctor Li Kegang proposed a method for solving the index weights by using the improved AHP method. The steps are described as follows:

(1) The simple correlation function is used to calculate correlation of stability class of the evaluation indexes. The correlation of an index to the evaluation class can be determined via the size of the correlation function. Bigger the class correlated with the index is, more unfavorable influence of this index on the system stability is;

(2) The maximal correlation and corresponding stability class of the evaluation indexes are identified;

(3) The indexes are sorted by significances by comparing the maximal correlation. If the correlation classes of $l$ indexes are same, all evaluation indexes can be sorted by analyzing and comparing the maximal correlation.

(4) The judgement matrix (table 3) based on nine-scale system (table 2) is constructed according to the previous index significance sorting.

The author analyzes sensitivity of the influence factors of the slope stability of the uniform road cutting by using the orthogonal design method and the analysis indexes include internal friction angle $\varphi$, cohesive strength $c$, slope height $H$, gradient $a$, rock soil volume weight $\gamma$, elastic modulus $E$, and Poisson ratio $\mu$. The sensitivities are sorted as $\varphi \geq H>c>a>\gamma>E>\mu$. The author thinks that it only indicates the static relation of the slope under influences of above several indexes. The factors affecting the slope stability are diversified in actual project. Some factors cannot be quantitatively analyzed. In a word, the author thinks that now the weight solving method proposed by the ISSN: 0010-8189 
Doctor Li Kegang is a relatively reasonable method.

Table 2 Judgement scale of index significance

\begin{tabular}{|c|c|}
\hline Scale $a_{i j}$ & Meaning \\
\hline 1 & The significance of $i^{\text {th }}$ factor is same as that of $j^{\text {th }}$ factor. \\
\hline 3 & The significance of $i^{\text {th }}$ factor is higher than that of $j^{\text {th }}$ factor. \\
\hline 5 & The significance of $i^{\text {th }}$ factor is much higher than that of $j^{\text {th }}$ factor. \\
\hline 7 & The significance of $i^{\text {th }}$ factor is critically higher than that of $j^{\text {th }}$ factor. \\
\hline 9 & The significance of $i^{\text {th }}$ factor is extremely higher than that of $j^{\text {th }}$ factor. \\
\hline 4 , 8 . & The significance of $i^{\text {th }}$ factor and $j^{\text {th }}$ factor is between the above classes. \\
\hline reciprocal of above numbers & If the comparison scale of $i$ and $j$ is $a_{i j}$, the comparison scale of $j$ and $i$ is $a_{j i}$. \\
\hline
\end{tabular}

Table 3 Judgment matrix

\begin{tabular}{|c|c|c|c|c|}
\hline Factor $c$ & $c_{1}$ & $c_{2}$ & $\cdots$ & $c_{n}$ \\
\hline$c_{1}$ & $b_{11}$ & $b_{12}$ & $\cdots$ & $b_{1 n}$ \\
\hline$c_{2}$ & $b_{21}$ & $b_{22}$ & $\cdots$ & $b_{2 n}$ \\
\hline$\ldots$ & $\ldots$ & $\cdots$ & $\cdots$ & $\cdots$ \\
\hline$c_{n}$ & $b_{n 1}$ & $b_{n 2}$ & $\cdots$ & $b_{n n}$ \\
\hline
\end{tabular}

(5) Calculate the corresponding vector of the maximal characteristic root of the judgement matrix in the table 3 , check the deviation consistence index $C I$ (equation 2), random consistence index $R I$ (table 4) and consistence proportion $C R(C R<0.1$ and a smaller value is better). If it passes check, after the feature vector is normalized, the weights of the indexes can be obtained. If it fails to pass check, the judgement matrix shall be constructed again and the maximal characteristic root and feature vector shall be calculated till it passes check.

$$
C I=\frac{\lambda_{\max }-n}{n-1}
$$

ISSN: 0010-8189 
In the equation (2):

$\lambda_{\max }-$ maximal characteristic root of the matrix;

$n$-Index number (namely matrix order).

Table 4 Random consistence index

\begin{tabular}{|c|c|c|c|c|c|c|c|c|c|c|c|c|}
\hline Matrix order & 1 & 2 & 3 & 4 & 5 & 6 & 7 & 8 & 9 & 10 & 11 & 12 \\
\hline$R I$ & 0 & 0 & 0.52 & 0.89 & 1.12 & 1.26 & 1.36 & 1.41 & 1.46 & 1.49 & 1.52 & 1.54 \\
& $C R=\frac{C I}{R I}$
\end{tabular}

3.3 Identify classical domain, node domain and matter-element under evaluation

(1) Classical domain

The classical domain is expressed with the matter-element as follows:

$$
M_{j}=\left(P_{j}, c_{i}, v_{i}\right)=\left[\begin{array}{ccc}
P_{j}, & c_{1}, & v_{j 1} \\
& c_{2}, & v_{j 2} \\
\vdots & \vdots \\
& c_{n}, & v_{j n}
\end{array}\right]=\left[\begin{array}{ccc}
P_{j}, & c_{1}, & <a_{j 1}, b_{j 1}> \\
& c_{2}, & <a_{j 2}, b_{j 2}> \\
\vdots & \vdots \\
& c_{n}, & <a_{j n}, b_{j n}>
\end{array}\right]
$$

(4)

In the equation (4):

$P_{j}$ - divided $j$ stability class;

$c_{i}-i^{\text {th }}$ evaluation index

$v_{j i}$-Value range of class $P_{j}$ on $c_{i}$, namely classical domain and $v_{j i}=\left\langle a_{j i}, b_{j i}\right\rangle$

(2) Node domain

The node domain is expressed with the element as follows:

ISSN: 0010-8189 


$$
M_{p}=\left(P, c_{i}, v_{p}\right)=\left[\begin{array}{ccc}
P, & c_{1}, & v_{p 1} \\
& c_{2}, & v_{p 2} \\
\vdots & \vdots \\
c_{n}, & v_{p n}
\end{array}\right]=\left[\begin{array}{ccc}
P, & c_{1}, & <a_{p 1}, b_{p 1}> \\
c_{2}, & <a_{p 2}, b_{p 2}> \\
\vdots & \vdots \\
c_{n}, & <a_{p n}, b_{p n}>
\end{array}\right]
$$

In the equation (5):

$P$-all evaluation classes;

$v_{p \mathrm{i}}$-Value range of $c_{i}$ under $P$ condition, namely the node domain of $P$ and $v_{p i}=\left\langle a_{p i}, b_{p i}\right\rangle, i$ is the evaluation index number $(i=1,2, \ldots, n)$.

The node domain is only one auxiliary expression and indicates the value range of the evaluation indexes. It can be referred in case of calculation and standardization of correlation.

(3) Matter-element under evaluation

The matter-element under evaluation is expressed as follows:

$$
M_{0}=\left[\begin{array}{ccc}
P_{0}, & c_{1}, & v_{1} \\
& c_{2}, & v_{2} \\
\vdots & \vdots \\
& c_{n}, & v_{n}
\end{array}\right]
$$

In the equation (6):

$P_{0}$ - things under evaluation (slope under analysis);

$v_{i}$-Measurement of $P_{0}$ o the index $c_{i}$, namely specific data of things under evaluation.

3.4 Identify the correlation $K_{j}\left(v_{i}\right)$ of the evaluation indexes on stability class

Based on actual conditions, the correlation function is selected when the optimal is located at and is not located at the middle point of the interval, or the simple correlation is selected and correlation of evaluation indexes to the stability classes are calculated.

3.5 Identify the correlation and evaluation class of matter-element under evaluation on the class $j$

For each feature $c_{i}$, the weight is $a_{i}$ and $\sum_{i=1}^{n} a_{i}=1$. Given $K_{j}\left(P_{0}\right)=\sum_{i=1}^{n} a_{i} K_{j}\left(v_{i}\right), K_{j}\left(P_{0}\right)$ is the correlation of the slope $P_{0}$ under evaluation on the class $j$. The corresponding stability class of the maximum of the $K_{j}\left(P_{0}\right)$ is the class of the slope stability. To easily compare and eliminate influences of the index value interval on the correlation function values, the correlation shall be converted the standardized correlation within $\langle-1,1\rangle$ according to the equation (1).

\section{Given}

ISSN: 0010-8189 


$$
\bar{K}_{j}\left(P_{0}\right)=\frac{K_{j}\left(P_{0}\right)-\min _{j} K_{j}\left(P_{0}\right)}{\max _{j} K_{j}\left(P_{0}\right)-\min _{j} K_{j}\left(P_{0}\right)},
$$

The stability class variant feature of the slope $P_{0}$ under evaluation is expressed as follows:

$$
j^{*}=\frac{\sum_{j=1}^{m} j \cdot \bar{K}_{j}\left(P_{0}\right)}{\sum_{j=1}^{m} \bar{K}_{j}\left(P_{0}\right)}
$$

\section{Discussions}

\subsection{Selection of correlation function}

Now the correlation functions established according to the Extenics theory include the correlation for optimal point at the middle point of the interval, correlation for optimal point not at the middle point of the interval and simple correlation function. When the slope stability is analyzed, the basic interval of an evaluation index is same. E.g. in the table 1, "slight stability" class corresponds to the evaluation index "crustal stress", which value is $\langle 2,8\rangle$ (unit: $\mathrm{MPa}$ ). If the value of the crustal stress exceeds this range, the evaluation index does not belong to "slight stability". In this case, the interval will be degenerated to single interval. On this occasion, the simple correlation function is used to express the compliance of the evaluation to stability class.

In the simple correlation function, the positive domain can be within the finite interval $X=\langle a, b\rangle$, or within the infinite interval $X=\langle a,+\infty)$, within infinite interval $X=(-\infty, b>$ and within the infinite interval $X=(-\infty,+\infty)$. In the table 1 , note the index value range in the first (stable) and final stability class (very stability). From the numeral expression in the table, when correlation of these indexes are calculated, it seems that the correlation function for the positive domain within the infinite interval $X=\langle a,+\infty)$ or within the infinite interval $X=(-\infty, b>$ is applicable, but the fact is not it. E.g. the actual values of the rock body quality index RQD and structural feature of the rock body are within $0 \sim 100$. Generally, the slope angle does not exceed $90^{\circ}$. The seismic intensity range regulated in China is within class 1-12. These indexes have specific maximum and minimum. The theoretical minimums of the crustal stress, cohesive strength, internal friction, slope height and daily maximal rainfall are 0 and the maximum cannot be determined, but they shall not be $+\infty$, therefore, it is not reasonable to calculate the correlation for the positive domain within the infinite interval for such evaluation indexes. A relative reasonable maximum can be regulated. The simple correlation function (equation 7) for the positive domain within the finite interval is used to calculate the correlation.

$$
k(x)=\left\{\begin{array}{l}
\frac{x-a}{M-a}, x \leq M \\
\frac{b-x}{b-M}, x \geq M
\end{array}\right.
$$

ISSN: 0010-8189 
The positive domain is within the finite interval $X=<a, b>$ in the equation (7) and the optimal point is expressed as $M \in X$.

For easy calculation, when the stability is evaluated for a slope, for indexes without specific maximum, when the corresponding index value of this slope exceeds the upper limit required by the "stability" and "very instability", this value can be used as the index maximum.

\subsection{Selection of optimal points}

After a correlation function is selected to calculate the correlation, the optimal points shall be identified. When the optimal point is within the positive domain of the correlation function, the value can satisfy the given conditions. The index value for satisfying an evaluation class is the optimal point of this index for this stability class in the value range of the stability class of the evaluation class regulated for the slope stability classification standard.

According to the meaning of the correlation function, the optimal points can be the middle point in the corresponding index value interval for three evaluation classes (slight stability, basic stability and no stability) in the table 1. E.g. if the value range of the corresponding evaluation class of the evaluation index "crustal stress" is $<14,20\rangle$, the optimal point shall be $17 \mathrm{MPa}$ at the middle of the interval, namely when the crustal stress is $17 \mathrm{MPa}$, it can satisfy "no stability" requirement well (at this time, the correlation function value is maximal). When the value is within 14-20 and the value is less than $17 \mathrm{MPa}$, it is thought as "no stability" class and tends to be "basic stability" (the correlation function value is smaller). When the value is more than $17 \mathrm{MPa}$, it is thought as "no stability", but it tends to be "very instability" (the correlation function value is smaller).

For the "stability" and "very stability" evaluation class, the optimal points shall be selected carefully. From the meaning of the optimal points, at this time, the optimal points shall be selected from the end of the corresponding value interval. Although such selection method will lead to mutation of the correlation function value, if an index has the maximal correlation for the "very instability" class, the correlation for other classes shall decrease, but the optimal point of this index for the corresponding "stability" class is selected from the interval end, the correlation of this index for "stability" class may be higher. Such inconsistent case is caused by unequal gaps of the optimal points of indexes for one evaluation classes and can be adjusted by calculating the standardized correlation. Therefore, for "stability" class, the right end of the corresponding index value interval shall be selected as the optimal point for the indexes which prefer to higher value. The left end of the corresponding index value interval shall be selected as the optimal point for the indexes which prefer to lower value. E.g. for the evaluation index "crustal stress", the value range of the evaluation class "stability" is $<0,2>$, the optimal points shall be selected as the left end $0 \mathrm{MPa}$ of the interval because this index prefers to lower value. if other points are selected as the optimal points, the lower crustal stress indicates higher stability. For the "very stability" class, the left point shall be selected as the optimal point for the indexes which prefer to higher value and the right point shall be selected as the optimal point for the indexes which prefer to lower value.

\section{Conclusion}

This paper presents a method to evaluate the stability of complex slope based on the calculation method of correlation in Extenics. The evaluation steps are described as follows: select the evaluation indexes of the slope stability, identify the classification standard for the slope stability, identify the weights of the evaluation indexes by using the improved AHP method, identify the classical domain, node domain and matter-element under evaluation, calculate the correlation of evaluation indexes for stability classes, standardize the correlation, and identify the correlation and evaluation class of the matter-elements for the class.

In analysis on the slope stability, the selected evaluation indexes shall highlight the control factors affecting the

ISSN: 0010-8189 
slope stability, namely it can completely show the natural and geological environment of the slope, can completely show the geometric features of the slope, can show the influences of the human activities on the slope stability, and ensure easy calculation and reasonable weight assignation.

In General, when the slope stability is evaluated, the simple correlation function is selected to express compliance of the evaluation index to the stability class. When the simple correlation function is used, the positive domain within the finite interval or infinite interval will be selected according to evaluation indexes. The theoretical minimums of some indexes is 0 . Although the maximum cannot be identified, but it shall not be $+\infty$. For such evaluation indexes, a relatively maximum shall be regulated manually and the simple correlation function for the positive domain within the finite interval is used to calculate the correlation.

For "stability" class, the right end of the corresponding index value interval shall be selected as the optimal point for the indexes which prefer to higher value and the left end of the corresponding index value interval shall be selected as the optimal point for the indexes which prefer to lower value. For "very stability" class, the left end of the corresponding index value interval shall be selected as the optimal point for the indexes which prefer to higher value and the right end of the corresponding index value interval shall be selected as the optimal point for the indexes which prefer to lower value.

Applying the calculation method of correlation in Extenics to evaluate the stability of complex slope has the advantages of intuitive evaluation process, good communicability, strong logic, and flexible selection of correlation function to calculate the correlation of evaluation index.

\section{Acknowledgements}

This research was supported by Scientific Research Fund Project of Yunnan Education Department (Grant No. 2021J0717) and Basic research project of Yunnan Province (Grant No. 202101AT070144).

\section{References}

[1] L. Wang, "Stability analysis of rock-mass slope based on extenics," Hebei Metallurgy, no. 1, pp. 21-23, 1999.

[2] Q.M. Xie, Y.Y. Xia, "Extension classification prediction method for the stability of rock-mass slopes," Chinese Journal of Rock Mechanics and Engineering, vol. 22, no.3, pp. 438-441, 2003.

[3] K.G. Li, J. Xu, S.C. Li, et al, "Research on evaluation of the slope stability based on the extension theory," Journal of Chongqing Jianzhu University, vol. 29, no. 4, pp. 75-78, 2007.

[4] K.G. Li, K.P. Hou, C.Q. Zhang, "Study on index weight of slope stability evaluation based on extensible simple dependent degree," Mining Research and Development, vol. 27, no. 6, pp. 24-16, 36, 2007.

[5] K.G. Li, K.P. Hou, W. Li, "Research on influences of factors dynamic weight on slope stability," Rock and Soil Mechanics, vol. 30, no. 2, pp.492-496, 2007.

[6] K.G. Li, "Study on Stability analysis and deformation prediction of rock slope," Chongqing University, 2006. 
[7] X.L. Tan, W.Y. Xu, G.L. Liang, “Application of extenics method to comprehensive safety evaluation of rock slope," Chinese Journal of Rock Mechanics and Engineering, vol. 28, no. 12, pp. 2503-2509, 2009.

[8] W. Cai, C.Y. Yang, "Basic theory and methodology on Extenics," Chin Sci Bull (Chin Ver), vol. 58, no. 13, pp. 1190-1199, 2013.

[9] Z.B. Peng, Z.M. He, W.X. Peng, et al. "Application of Fuzzy Judging on Rock slop Stability Analysis,” Mining and Metallurgical Engineering, vol. 25, no. 3, pp. 1-4, 2005.

[10] J.Q. Zhou, C. Tang, "The model of fuzzy comprehensive judgement applied in evaluations of slope stability,” Journal of Natural Disasters, vol. 4, no. 3, pp. 73-82, 1995.

[11] H.S. Ma, L.X. Zheng, "Quantitative analysis of influence factors of slope stability by using neural network algorithm," Subgrade Engineering, no. 5, pp. 42-45, 2005.

[12] S.D. He, T. Qu, X.H. Huang, "Improved prediction method based on feedforward neural network and its application in landslide stability prediction,” Forecasting, no. 3, pp. 61-63, 1997.

[13] Y.C. Zhai, W.G. Cao, J.Y. Wang, et al. "Fuzzy evaluation method of slope stability based on uncertain analytic hierarchy process," Rock and Soil Mechanics, vol. 32, no. S2, pp. 539-543, 2011.

[14] Y.Y. Liu, Y.Q. Li, Z.Z. Guo, et al. "Research on risk evaluation of highway slope based on AHP-Fuzzy and laser point cloud data," Journal of Catastrophology, vol. 33, no. 1, pp. 206-212, 2018.

[15] S.G. Du, "Method of equal accuracy assessment for the stability analysis of large open-pit mine slopes," Chinese Journal of Rock Mechanics and Engineering, vol. 37, no. 6, pp. 1301-1331, 2018.

[16] X.G. Dai, B. Zhang, Z.Z. Zheng, "Application of finite cloud model and distance discrimination in slope stability evaluation,” Journal of Railway science and Engineering, vol. 15, no. 1, pp. 71-78, 2018.

[17] J.X. Wang, Z.H. Zhou, B. Fu, et al. "Application of factor analysis and probabilistic neural network model on evaluation of the slope stability," Hydrogeology \& Engineering Geology, vol. 45, no. 2, pp. 123-130, 2018.

[18] D.L. Liu, G.H. Tan, Q.G. Li, et al. "The stability of rock slope and fuzzy comprehensive evaluation method," Chinese Journal of Rock Mechanics and Engineering, vol. 18, no. 2, pp. 170-175, 1999.

[19] M.Y. Liu, "Intelligent approach to slope stability evaluation based on case-based reasoning," Wuhan University of Technology, 2001. 